\title{
CHARACTERIZATION OF THE COHERENCE PROPERTIES OF DIFFERENT OPTICAL SOURCES
}

\author{
Celine Kavida. $\mathbf{A}^{1,2}$ and A. Roberts ${ }^{1}$ \\ ${ }^{1}$ School of Physics, The University of Melbourne, \\ Melbourne, Victoria, Australia \\ ${ }^{2}$ Vel Tech Multi Tech Dr. Rangarajan Dr. Sakunthala Engineering \\ College, Avadi, Chennai-600062, Tamil Nadu, India \\ e-mail: celinearuldoss@gmail.com
}

\begin{abstract}
Non-interferometric phase-space tomography is used to characterize the spatial coherence properties of scalar quasi-monochromatic partially coherent optical fields. Three sources were investigated and characteristic projections through the correlation functions determined. It was seen that a single-mode fiber-coupled source exhibited a much longer coherence length than a multi-mode fibre-coupled source. Both sources exhibited a high degree of symmetry. A collimated light emitted diode, on the other hand, was shown to have a short spatial coherence length and clear asymmetries were evident.
\end{abstract}

Keywords: Optics, Photonics, Coherence

\section{INTRODUCTION}

Quasi-monochromatic, scalar, partially coherent wavefields are completely characterized by one of the correlation functions. The mutual optical intensity $G\left(\underset{r_{1}}{r_{2}} r_{2}\right)$ can be defined as

$$
G\left(\stackrel{\mathrm{r}}{r_{1}}, r_{2}\right)=\left\langle E\left(\stackrel{\mathrm{r}}{r_{1}}, t\right) E^{*}\left(\stackrel{r}{r_{2}}, t\right)\right\rangle
$$

Where the brackets \langle\rangle denote averaging over all realizations of the field. This function can also be expressed in terms of the position coordinates $r_{1}^{1}$ and $r_{2}$ and for later reference the function called the spatial coherence function $J(\underset{r}{r} \stackrel{r}{r})$ is defined in terms of the mean $\left(\stackrel{r}{r}=\frac{\stackrel{r}{r_{1}}+\stackrel{r}{r}}{2}\right)$ and the difference coordinate $\left(\stackrel{r}{s}=\stackrel{r}{r}{ }_{1}-\stackrel{r}{r} r_{2}\right)$ as

$$
J(\stackrel{\mathrm{r}}{r}, \stackrel{\mathrm{r}}{s})=G(\stackrel{\mathrm{r}}{r}+\stackrel{\mathrm{r}}{s} / 2, \stackrel{\mathrm{r}}{r}-\stackrel{\mathrm{r}}{s} / 2)
$$

Equivalently, the field can be described using a phase space representation called the generalised radiance or Wigner distribution function or phase-space density which is written as: 


$$
B(\stackrel{\mathrm{r}}{r}, \stackrel{\mathrm{r}}{u})=\int J(\stackrel{\mathrm{r}}{r}, \stackrel{\mathrm{r}}{s}) \exp (-i \stackrel{\mathrm{r}}{\mathrm{r}} \bullet \stackrel{\mathrm{r}}{s}) d \stackrel{\mathrm{r}}{s}
$$

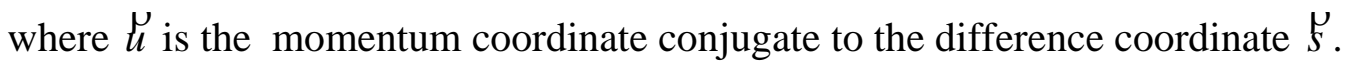

Historically, spatial coherence measurements have been determined using two-slit configurations [1] or interferemetric methods [2-6]. A single two-slit measurement can provide information about the correlations within a field between only two points in the field. In the case of fields that can be assumed to be statistically homogeneous, uniformly redundant arrays [7] can be used to determine the extent of correlations between points of different separation within the field. Interference techniques [8-6] can be used fully reconstruct the correlation function of interest. Such techniques, however, require more complex instrumentation than non-interferometric methods, can possess a relatively low numerical aperture and are challenged by sources with short coherence lengths where the fringe visibility can become extremely weak. In the previous decade a number of more sophisticated non-interferometic methods [8-11] have been proposed and demonstrated. Tu and Tamura [10] introduced a new method relating the intensity distribution to the correlation functions. Following this theory Dragoman et al.[12,13] performed phase space tomography (PST) to obtain the one-dimensional (1D) field of coherent sources including He-Ne [12] and Vertical Cavity Surface-Emitting Lasers [13]. PST has been applied not only in optics but also to the study of electron beams [14] and the beams of helium atoms [15,16]. A. Singer et al determined the coherence functions of the free electron Laser [17] and X-ray Synchrotron sources [18] using the Young's Double slit experiment. In a previous paper [19], we have shown that it is possible to reconstruct characteristic projections through the correlation functions by using only the simple propagation-based method originally proposed by Nugent [8]. Although such a method cannot fully characterize an arbitrary field, it can be used to determine projections. In the case of fields possessing a high degree of symmetry, or fields that are separable, this is sufficient to completely reconstruct the correlation functions. In general, however, it remains a useful tool in extracting information about arbitrary partially coherent fields. The theory of correlation functions based on wave transport model for the scattering imaging was made possible [20]. The reduction of speckle, improvement and the effect of spatial coherence length on the resolution of the bio-images were made possible by the low-coherence optical sources [21].

The decrease in coherence of a field due to atmospheric turbulence has been studied by various researchers [22\&23]. Knowledge of the propagation of a partially coherent field in a turbulent atmosphere is necessary in techniques such as long distance free space optical communications. Fei Wang et. al., [24\&25] has revealed a source which has a efficient data 
transfer with a fractional multi-Gaussian-correlated Schell-model compared to Gaussion Schell Model and studied the intensity correlations of the atmospheric turbulent fields of optical communication. Unlike insignificant effects on the partial coherence due to atmospheric turbulence the inhomogeneities in cases like biological tissues are found to be large. The decrease in the degree of coherence has been shown to influence the quality of biological imaging methods [26].

In this paper we discuss the application of non-interferometric PST to the characterization of scalar, quasi-monochromatic sources with varying degrees of spatial coherence. We use a simple formulation of PST which is based on assuming the sources depend on only one transverse spatial coordinate which permits the reconstruction of characteristic projections [8] through both the spatial coherence function and the generalized radiance. We show that, as expected, a source coupled to a single mode fibre possesses a relatively long coherence length compared to one attached to a multimode fibre. Furthermore, we examined a light emitting diode and saw that it has a substantially shorter spatial coherence length than the fibre coupled sources.

\section{EXPERIMENT}

The sources studied here were (i) a bandpass filtered LED, (ii) a bandpass filtered white light source (Mikropack, MODEL: HL-2000-FHSA) coupled to a multimode fibre and (iii) single-mode fibre-coupled SLD (Superlum Ltd. MODEL: 505). The field radiated by the LED source was passed through a bandpass filter with its transmission peak centered on $665 \mathrm{~nm}$ and with a passband of $10 \mathrm{~nm}$ to obtain a quasi-monochromatic field. In the case of the white light source, a filter centered on a wavelength of $521 \mathrm{~nm}$ and bandwidth of $10 \mathrm{~nm}$ was used to obtain a quasi-monochromatic wavefield. In the case of the multimode fibre-coupled source, a fibre collimating lens (Thorlabs Part number F260FC-B) was coupled with the fibre. This lens has an NA of 0.16 and focal length of $15.3 \mathrm{~mm}$. The fibre collimating lens approximately collimates the light rays of the field transmitted through the fibre. In the case of the singlemode fibre-coupled SLD, a $681 \mathrm{~nm}$ collimated beam was produced using an objective lens (Nikon, Japan) of magnification $\times 20$. Note that this changes the correlation functions compared to those of the field at the fibre output face.

The source field is transmitted through the achromatic meniscus lens (Melles Griot 09LAM229) of focal length $13.89 \mathrm{~cm}$ and the intensity distribution is recorded along the $z$ direction as shown in the Fig 1 . The numerical aperture (NA) of the lens is $\sim 0.1$. The intensity distributions of the LED source field were measured along the $z$-direction of the optical axis using the experimental set-up illustrated in Fig 1. Intensity distributions were recorded using a 
CCD camera (Roper Scientific-16 bit array). The size of the chip in the CCD camera is 1340 $\times 1300$ pixels with a pixel size of $20 \mu \mathrm{m}$. The camera, placed on a motorized translation stage (Coherent Scientific Serial No: ATS 62125) of length $1250 \mathrm{~mm}$ recorded the images at regular intervals. The stage has a high precision of $\pm 1 \mu \mathrm{m} / 25 \mathrm{~mm}$ and is controlled by a controller (Soloist20) via a personal computer. The shutter size of the CCD camera is $40 \mathrm{~mm}$ in length and height and the closing and opening time is 56 msec. This sets a lower bound on the exposure time. The images were recorded for a fixed exposure time for each complete experiment and the temperature was maintained at $-20^{\circ}$ Celsius. This low temperature for the camera was used to reduce the background noise. The temperature and the exposure time were maintained to be the same throughout the experiment to avoid fluctuations in the intensity measured along the optical axis.

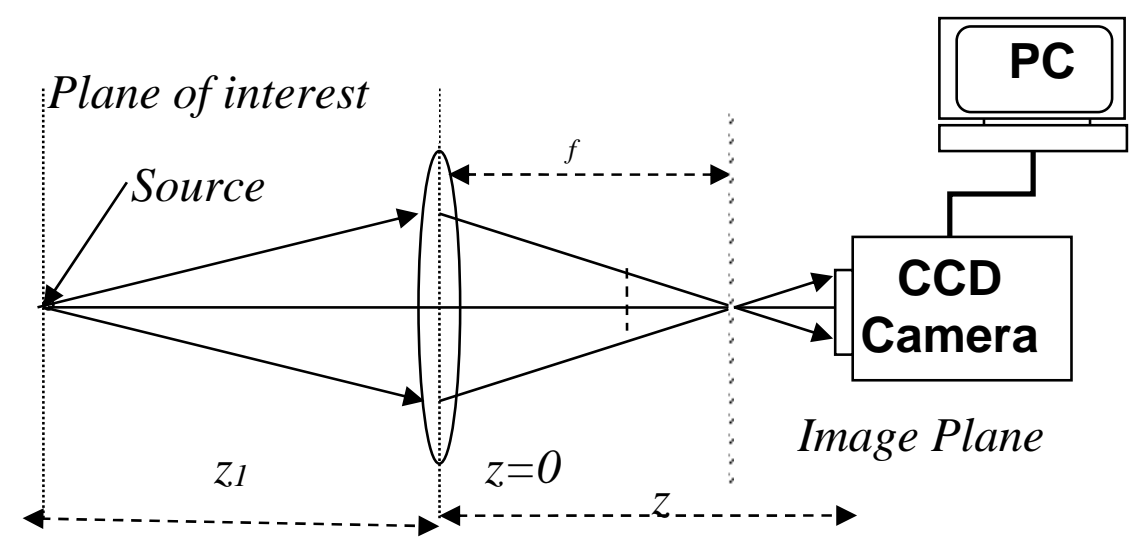

Fig. 1. Measurement of Intensity distribution following the Phase-Space Tomography method.

The distance from the lens of focal length $f$ at which we choose to reconstruct the generalized radiance is represented as $z_{1}$ (See Fig 1). A neutral density filter was positioned between the source and the focusing lens to avoid saturation of the camera chip at the focal point along the propagation z-direction in the case of the fibre-coupled SLD source.

The projections of the intensity distribution along the $x$-direction were calculated by summing all the pixels along the $y$-direction for all measured $z$-values. Similarly the projection can be obtained across any other direction such as the $x$-direction. Using this information for all $z$-values, projections through correlation functions of the two-dimensional field can be fully reconstructed along both the directions.

\section{RESULTS}

Following the theory explained in paper [17], the projections of the correlation functions of the field for different sources were reconstructed. The projections across the $x$ 
and $y$-coordinates of the spatial coherence functions $J^{(1)}\left(x, s_{x}, 0\right)$ and $J^{(2)}\left(y, s_{y}, 0\right)$ of the LED source with a wavelength of $665 \mathrm{~nm}$ and bandpass of $10 \mathrm{~nm}$, collimated fibre-coupled multimode white light source in front of the fibre collimating lens and of the collimated field produced by the single-mode fibre-coupled SLD at the exit of the objective lens were reconstructed and are shown in Fig 2 (a), (b) and (c) respectively.
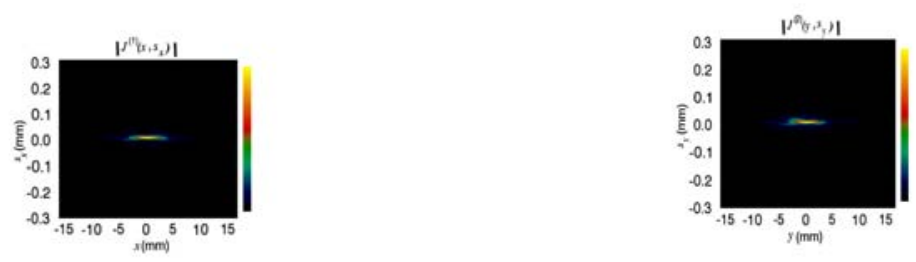

(a)
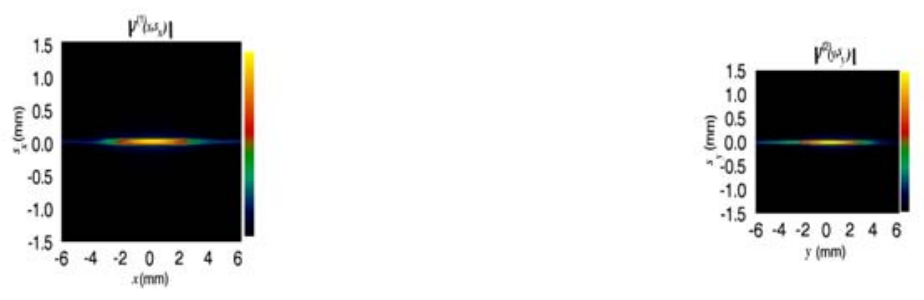

(b)
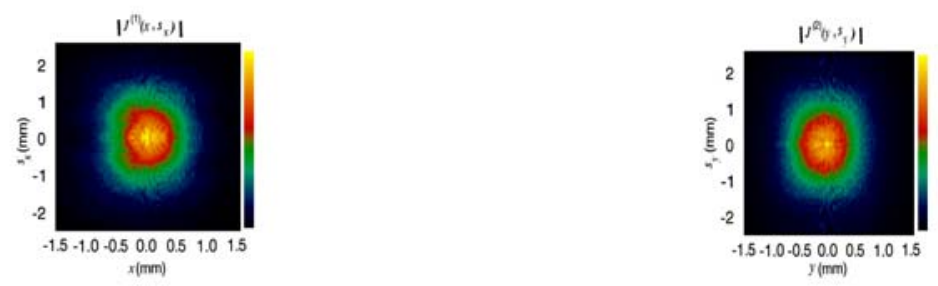

(c)

Fig 2 The experimentally reconstructed projection of the spatial coherence functions nm along (Left) $x$-direction $\boldsymbol{J}^{(1)}\left(x, s_{x}, 0\right)$ and (right) $y$-direction $\boldsymbol{J}^{(2)}\left(y, s_{y}, 0\right)$ of two-dimensional field (a) at the output face of the LED source peaked with a wavelength of $665 \pm 10 \mathrm{~nm}$ and bandpass of 10, (b) at the output face of the fibre collimating lens of the filtered multi-mode fibre-coupled white (white) light source with centre wavelength $521 \mathrm{~nm}$ and (c) collimated 
intensity field of the single-mode fibre-coupled SLD source of wavelength $681 \mathrm{~nm}$ in front of the objective lens.

Likewise the projections of the generalized radiance $B^{(1)}\left(x, u_{x}, 0\right)$ and $B^{(2)}\left(y, u_{y}, 0\right)$ of the fields of the above mentioned three sources were reconstructed and are shown in Fig 3 (a), (b) and (c).
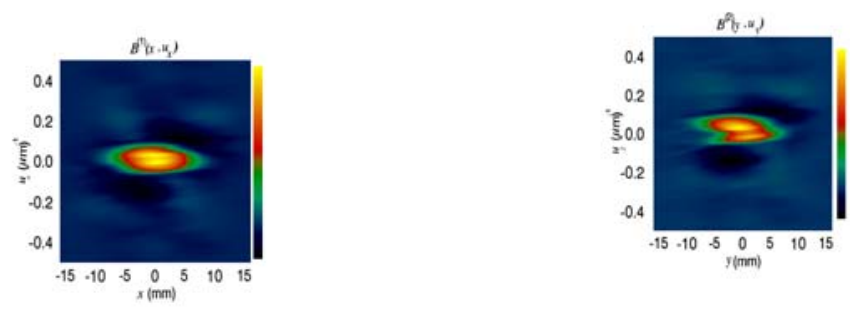

(a)
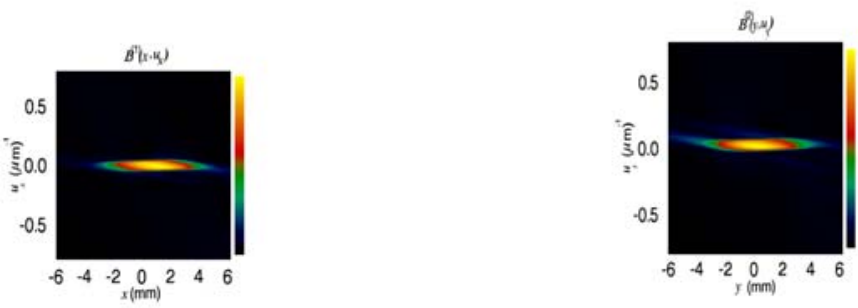

(b)
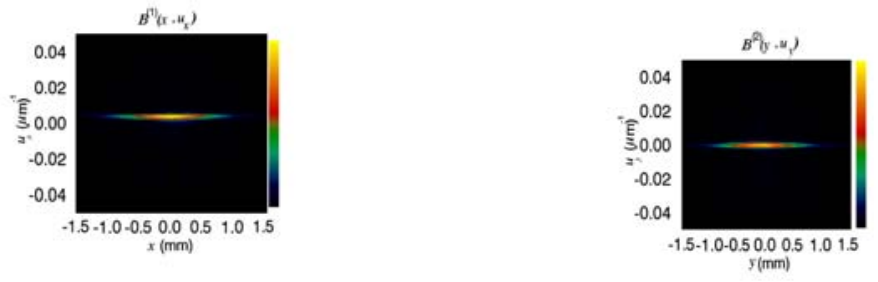

(c)

Fig 13 The experimentally reconstructed projection of the generalized radiance nm along (Left) $x$-direction $B^{(1)}\left(x, \boldsymbol{u}_{x}, 0\right)$ and (right) $y$-direction $B^{(2)}\left(y, \boldsymbol{u}_{y}, 0\right)$ of two-dimensional field (a) at the output face of the LED source peaked with a wavelength of $665 \pm 10 \mathrm{~nm}$ and bandpass of 10, (b) at the output face of the fibre collimating lens of the filtered multi-mode fibre-coupled 
white (white) light source with centre wavelength 521nm and (c) collimated intensity field of the single-mode fibre-coupled SLD source of wavelength $681 \mathrm{~nm}$ in front of the objective lens.

\section{DISCUSSION}

Table 1 Coherence lengths of different sources.

\begin{tabular}{|c|c|c|}
\hline \multirow{2}{*}{ Source } & \multicolumn{2}{|c|}{$\begin{array}{c}\text { Coherence length } \\
\text { (mm) }\end{array}$} \\
\cline { 2 - 3 } & $l_{x}$ & $l_{y}$ \\
\hline LED & $0.011 \pm 0.001$ & $0.009 \pm 0.001$ \\
\hline Multi-mode white light & $0.059 \pm 0.001$ & $0.058 \pm 0.001$ \\
\hline Single-mode collimate SLD & $1.95 \pm 0.02$ & $2.10 \pm 0.02$ \\
\hline
\end{tabular}

It can be easily seen in Fig 2 (a) that the correlations existing between the points in the field decreases rapidly as the magnitude of the coordinate $s$ in the spatial coherence function increases due to the low coherence nature of the LED source. Correspondingly the generalized radiance is broader along the angular coordinate $u$ in the phase space representation as shown in Fig 3 (a). It can be seen from Fig 3 (a) the reconstructed projections of the generalised radiance are not identical which reveals that the LED is not a symmetric source.

The quasi-monochromatic fibre-coupled multi-mode white light source was found to be more coherent by observing the nature of the correlation functions in comparison with the LED source as expected. This is apparent in the broader nature of the spatial coherence function along the separation coordinate $s$ in Fig 2 (b) and the corresponding narrower width of the generalized radiance along the angular coordinate $u$ in Fig 3 (b).

The coherence nature of the source is clearer with a profile plotted along the separation coordinate $s$ at $x=0$ along the normalized spatial coherence function as explained hereafter. The complex degree of coherence function can be defined as

$$
\gamma(\rho, \rho)=\frac{J(r, s)}{\sqrt{J(\rho / 2,0) J(-\xi / 2,0)}}
$$

This function normalizes the spatial coherence function to the intensity and provides information about the coherence alone. A Gaussian was fitted to the profile along the 
separation coordinate $s$ of $|\gamma|$ at $r=0$ using the function GAUSSFIT in the IDL programming language. The 1/e half width of the maximum of the profile along the separation coordinate $s$ of the absolute value of the degree of coherence gives a measure of the coherence length of the field. Thus the coherence length of the LED, multi-mode and single mode sources along both directions ( $l_{x}$ and $l_{y}$ ) were found.

Table 1 summarizes the coherence lengths of the LED, multi-mode fibre-coupled white light source and the single-mode fibre-coupled source along the both $x$ and $y$ projections of the correlation functions. The associated error is the standard deviation of the profile of the data obtained experimentally from the fit. The similarity of the spatial coherence values along both the directions reveals the spatial homogeneity property of the sources.

By comparing broader nature of the spatial coherence function along the separation coordinate $s$ of the LED (See Fig 2), the multi-mode fibre-coupled white light source (See Fig 2) and the single-mode fibre-coupled SLD (See Fig 2) source (Note the scaling difference) it is evident that the single-mode fibre-coupled source has a longer coherence length since it is apparent that correlations exists to longer distances for the SLD as depicted in Table 1.

The projections of the generalised radiance along both the directions $\left(B^{(1)}\left(x, u_{x}, 0\right)\right.$ and $\left.B^{(2)}\left(y, u_{y}, 0\right)\right)$ are in very close agreement and reveal the axially symmetric nature of the multimode white light source and single-mode fibre-coupled SLD source shown in Fig 3 (b) and (c) respectively.

\section{CONCLUSION}

This paper provides a detailed description of the non-interferometric phase-space tomographic experiment. Utilizing this method the correlation functions, the generalized radiance and the spatial coherence function, were reconstructed and compared for various sources with quite different coherence properties.

This non-interferometric method is straightforward to implement unlike other methods involving slits or uniformly redundant arrays and provides a measure of the spatial coherence at each point in the field for all slit separations and is readily extended to non-visible optical sources such as x-rays. The results of the experiments prove that the method is a relatively straightforward way for studying the coherence properties of range of sources. 


\section{ACKNOWLEDGEMENT}

This research was supported under Australian Research Council's Discovery Projects funding scheme (project number DP0557505). The authors would like to thank Nicoleta Dragomir, Chanh Tran, Keith Nugent and Rob Scholten for useful discussions.

\section{REFERENCES}

1. Y. Takayama, R. Z. Tai, T. Hatano, T. Miyahara, W. Okamoto and Y. Kagoshima. J. Syn. Rad., 5, 456-458 (1998).

2. C. K. Hitzenberger. J. Mod. Opt., 46, 1763-1774 (1999).

3. C. Iaconis and I. A. Walmsley. Opt. Lett., 21, 1783-1785 (1996).

4. A. Wax and J. E. Thomas. Opt. Lett., 21, 1427-1429 (1996).

5. K. Vogel and H. Risken. Phys. Rev. A, 40, 2847 (1989).

6. C.-C. Cheng and M. G. Raymer, J. Mod. Opt. 47, 1237-1246 (2000)

7. J. J. A. Lin, D. Paterson, A. G. Peele, P. J. McMahon, C. T. Chantler, and K. A. Nugent, Phys. Rev. Lett. 90, 074801 (2003).

8. K. A. Nugent, Phys. Rev. Lett. 68, 2261-2264 (1992).

9. M. G. Raymer, M. Beck and D. F. McAlister.Phys. Rev. Lett., 72, 1137-1140 (1994).

10. J. Tu and S. Tamura. Phys. Rev. E, 55, 1946-1949 (1997).

11. G. Hazak. Phys. Rev. Lett. 69, 2874 (1992).

12. D. Dragoman, M. Dragoman and K.-H. Brenner. App. Opt., 41, 5512-5518 (2002).

13. D. Dragoman, M. Dragoman and K. H. Brenner. Opt. Lett., 27, 1519-1521 (2002).

14. K. Chalut, V. N. Litvinenko and I. V. Pinayev. Phys. Rev. Special Topics: Acc. and Beams, 8, 102802 (2005).

15. U. Janicke and M. Wilkens. J. Mod. Opt., 42, 2183-2199 (1995).

16. T. Pfau and C. Kurtsiefer. J. Mod. Opt., 44, 2551-2564 (1997).

17. Asinger, I. A. Vartanyants, M. Kuhlmann, S. Duesterer, R. Treusch, and J. Feld-haus..Phys. Rev. Lett.101, 254801 (2008).

18. A. Vartanyants and A. Singer.New Journal of Physics 12 , 035004 (2010).

19. C. K. Aruldoss, N. M. Dragomir, and A. Roberts.J. Opt. Soc. Am. A 24, 3189-3197 (2007).

20. C. K. Aruldoss. \& Roberts. Opt. Exp., 16, 26, 21389- 21400 Dec (2008).

21. Azeem Ahmad, Vishal Srivastava, Vishesh Dubey, and D. S. Mehta, Applied Physics Letters, 106,093701 (2015).

22. G. Gbur and W. Wolf, J. Opt. Soc. Am. A 19, 1592-1598 (2002).

23. Dogariu and Amarende, Opt. Lett., 28, 10-12 (2003).

24. Fei Wang, Italo Toselli, Jia Li and Olga Koratkova, Opt. Lett., 42, 6, 1129-1132, (2017)

25. Jia Li, Fei Wang and Olga Koratkova, Opt. Exp., 24, 15, 17779-1779, (2016).

26. C. C. Chengg and M. G. Raymer, Phys. Rev. A, 62, 023811-1-023811-12 (2000). 
